\title{
Experiences and Conclusions of the Hungarian Emergency Sepsis Register
}

\author{
Péter Kanizsai1, Tamás Berényi1 ${ }^{1}$ Miklós Pálvölgyi², János Gál ${ }^{3}$, Zoltán Pető ${ }^{4}$ \\ ${ }^{1}$ Department of Emergency Medicine, Clinical Centre, Semmelweis University, Budapest, Hungary \\ ${ }^{2}$ Department of Social Sciences, Faculty of Health Sciences, Semmelweis University, Budapest, Hungary \\ ${ }^{3}$ Department of Anaesthesia and Intensive Care, Division of Emergency Medicine and Oxyology, Faculty of Medicine, \\ Semmelweis University, Budapest, Hungary \\ ${ }^{4}$ Department of Emergency Medicine, Szent-Györgyi Science University, Faculty of Medicine, Szeged, Hungary \\ Email:plkanizsai@yahoo.com
}

How to cite this paper: Kanizsai, P., Berényi, T., Pálvölgyi, M., Gál, J. and Pető, Z. (2017) Experiences and Conclusions of the Hungarian Emergency Sepsis Register. Open Journal of Epidemiology, 7, 44-58. https://doi.org/10.4236/ojepi.2017.71005

Received: December 6, 2016

Accepted: February 6, 2017

Published: February 9, 2017

Copyright (c) 2017 by authors and Scientific Research Publishing Inc. This work is licensed under the Creative Commons Attribution International License (CC BY 4.0).

http://creativecommons.org/licenses/by/4.0/

\section{cc) (7) Open Access}

\begin{abstract}
Objective: Early recognition is a key step in decreasing morbidity and mortality in sepsis. Like in other European countries an ever growing number of septic patients enter hospitals via emergency departments. Our goal was to assess the number and severity of patients with sepsis, severe sepsis and septic shock attending the Hungarian emergency departments. Methods: Twohundred and ninety-nine patients were entered into the Hungarian Emergency Sepsis Register, focusing on age, disease severity, monitoring and oxygenation. Application and time of administration of antibiotics, use of biomarkers, i.e. lactate and procalcitonin, disposition of patients were assessed using a webbased electronic questionnaire. Results: Data arriving from twenty emergency departments revealed that with severity of the disease oxygen administration and level of monitoring increased, along with antibiotic administration and use of biomarkers. Time spent in the ED did not differ between septic, severely septic and septic shock patients; however higher level of care was provided for the sickest patients. Conclusion: Our register data indicate that sepsis presents a substantial challenge to Hungarian emergency departments. The set standards for timely, adequate treatment are not yet met. The results are however also influenced by charactersitics of the Hungarian health care system, most notably the lack of hospice systems. Further studies are needed to separate the subset of patients who would require and of life care.
\end{abstract}

\section{Keywords}

Sepsis, Register, Monitoring, Antibiotic, Disposition, Lactate, Procalcitonin

\section{Introduction}

Since the recognition of the germ theory by the Hungarian physician Semmel- 
weis, sepsis has been acknowledged as a severe and increasingly important issue worldwide. It has been in the focus of attention of numerous professional organisations [1] and there is an increasing awareness regarding early recognition and early treatment [2] [3].

Sepsis has become a global problem with its high mortality and increasing incidence [4]. The disease has been classified according to its severity, i.e. sepsis, severe sepsis and septic shock developing frequently to multiorgan failure regardless of the advances in sepsis care [5]. Despite all efforts, the morbidity and mortality of sepsis in Europe have not decreased in a desired manner [6].

The introduction of early goal directed therapy [7] has established the firm basis of a paradigm shift in treating septic patients in the emergency department (ED). With a great deal of turbulence with the theory and practice of early goal directed therapy new data have emerged regarding the targeted parameters [8] [9] but it is still agreed that therapy should be based on optimal oxygenation, fluid replacement, antimicrobial agent and eradication of the source of sepsis. It is also prudent to perform microbiological sampling ideally before the administration of the antimicrobial agent [3].

A new approach has emerged in the timely management of sepsis in the emergency departments by introducing the "Sepsis Six" method. This encounters six cornerstones of recognition and treatment by delivering appropriate amount of oxygen, obtaining blood cultures, administering empiric but wide spectrum antibiotics, measuring serum lactate, starting intravenous fluids and measuring urine output [10] [11] [12].

One of the reasons that sepsis is responsible for so many deaths and disabilities is that diagnosis is far from straightforward. If one suffers a fracture, it can be visualized by using an X-ray and it is fairly simple to make a decision on further treatment. However, sepsis has no specific imaging or laboratory findings. According to the Surviving Sepsis Campaign guidelines we are required to make a diagnosis on the basis of general variables (fever, hypothermia, tachycardia, tachypnea, hyperglycaemia, peripheral oedema), inflammatory variables (leukocytosis, leukopenia, normal white-cell count with $>10 \%$ immature forms, elevated plasma C-reactive protein (CRP), elevated plasma procalcitonin (PCT)), haemodynamic variables (arterial hypotension, or decrease in systolic pressure of $>40 \mathrm{mmHg}$ in adults, elevated mixed venous oxygen saturation, elevated cardiac index), organ-dysfunction variables (arterial hypoxemia, acute oliguria, increased creatinine levels, coagulation abnormalities, paralytic ileus, thrombocytopenia, hyperbilirubinemia) and tissue-perfusion variables (hyperlactataemia, decreased capillary refill or mottling) [3].

One can quickly recognize that none of these variables are specific for sepsis, and this implicates that it is prudent to be vigilant if patients with the above signs and symptoms are showing up in the emergency department. A high level of suspicion must be maintained regarding sepsis, because delay, especially in more severe forms of sepsis can dramatically increase mortality. It should also be noted that septic patients must be assessed in a complex way, considering pre- 
disposition, immune status and comorbidities to get a picture as clear as possible on the septic processes.

It is also important to assess our patients in terms of sepsis severity. Progression from sepsis to severe sepsis or septic shock is a continuum; however it is always more challenging to treat a patient with an advanced septic state. In severely septic patients delay in administration of antibiotics increased mortality by $7.6 \%$ each hour [13].

Recently the SEPSIS-3 task force suggested a new approach and nomenclature for septic patients [14]. According to the new recommendations septicaemia, severe sepsis and sepsis syndrome are eliminated [15] which leaves us with the categories of sepsis and septic shock. Organ dysfunction is defined on the basis of a quick SOFA score (Table 1), i.e. altered mental state with new onset resulting in a Glasgow Coma Scale less than 15, a respiratory rate higher than 22/min and a systolic blood pressure lower than $100 \mathrm{mmHg}$. If a patient scores two or more than the suspicion of sepsis is confirmed by the SOFA score using $\mathrm{PaO}_{2} /$ $\mathrm{FiO}_{2}$ ratio, mean arterial pressure, urine output or creatinine levels, serum levels of bilirubin, the GCS, platelet count and need of vasoactive support. Sepsis is defined if SOFA score is 2 or higher than that.

Regardless of any changes in classification, it is well recognized that time-dependent management of sepsis is a key to better survival. Therefore we were keen to get an accurate picture on the recognition, triage, early treatment and disposition of the septic patients presenting in the Hungarian emergency departments over a two year period and also wanted to follow their clinical pathways.

\section{Methods}

An online survey was constructed using the webspace of the Hungarian Association of Emergency Medicine (www.msotke.hu) after the ethical committee was approached for the need of any permission to carry out the survey. The committee decided to waive the consent since patient identification was impossible and the answers of the survey did not affect the treatment of any patient.

Patient selection was based on the presenting symptoms, using the criteria established in the 2013 Surviving Sepsis Guidelines [6], but suspicion and/or presence of an infection was the key determinant in making the diagnosis. Sepsis was defined as a documented, or suspected infection with general, inflammatory, haemodynamic, tissue perfusion and organ dysfunction variables. Septic shock was identified as sepsis-induced tissue hypoperfusion or organ dysfunction, while septic shock was the diagnosis if there was a hypotension and tissue hypoperfusion in severely septic patients despite adequate fluid therapy. In the data

Table 1. The quick SOFA score. A score of two or more raises the suspicion of sepsis.

\begin{tabular}{c} 
Parameter \\
\hline Altered mental state GCS $<15$ \\
Respiratory rate $>22 /$ minute \\
Systolic blood pressure $<110 \mathrm{mmHg}$ \\
\hline
\end{tabular}


analysis any patient, older than 18 years of age was recruited who met the criteria of sepsis, severe sepsis and septic shock and was admitted to the emergency department.

Anyone wishing to send data could log in with his/her login name and password. The questionnaire was anonymous with questions on admission time (yyyy.mm.dd hh:min), gender and age of the patient, severity of sepsis, i.e. sepsis, severe sepsis or septic shock, use of lactate and PCT as biomarkers of sepsis, time of microbiological sampling, time of antibiotic administration, oxygen administration, need for mechanical ventilation, need for invasive monitoring, administration of a vasoactive agent and disposition of the patient.

The decision on the three categories were at the discretion of the physician caring for the patient and was made on the basis of the definitions established in the Surviving Sepsis Campaign recommendations but not registered in the questionnaire.

Data collection was over a period of 18 months. Twenty different hospitals with fully equipped and staffed emergency departments participated in the study.

Anonymous data were stored on a secure server and only one investigator (P.K.) had access to them up to the point of statistical analysis when the statistician (M.P.) was also authorised for data usage.

Statistical analysis was carried out using Kolmogorov-Smirnov and ShapiroWilk test to assess normality, the intragroup deviance was tested by Levene test. According to the results of pretests parametric data were examined by Anova and non-parametric by Kruskal-Wallis tests.

\section{Results}

The data of two-hundred and ninety-nine patients were analysed. Patients were divided into three groups according to the severity of the sepsis: septic (Sep), severely septic (SeSep) and those in septic shock (SepSh). One-hundred and fourty-two patients (47\%) fell into the septic, 77 into the severely septic (26\%) and 80 into the septic shock (27\%) group (Figure 1). The number of patients in the septic group was significantly higher than those comprising the severely septic and septic shock groups (Figure 2). The average age of the patients in Sep, SeSep and SepSh groups were 66 (CI 3.15), 69.8 (CI 3.85), 71.9 (CI 2.49) years respectively, showing significantly younger patients in the sepsis group compared to the others (Figure 3). The gender of patients did not differ significantly, there were 72 male, 70 female patients in the septic group, 46 male and 49 female in the severely septic along with 43 male patients and 37 female patients in the septic shock group (Table 2).

Additional oxygen was administered in 69 (48.6\%) of the septic patients, 58 (75.3\%) in the severely septic and 78 (97.5\%) in those with septic shock, showing significant difference in the two more severe groups compared to the group of septic patients (Figure 4).

Apart from routine ECG, blood pressure, temperature and percutaneous oxygen saturation measurements invasive monitoring was initiated only in $1.4 \%$ of 


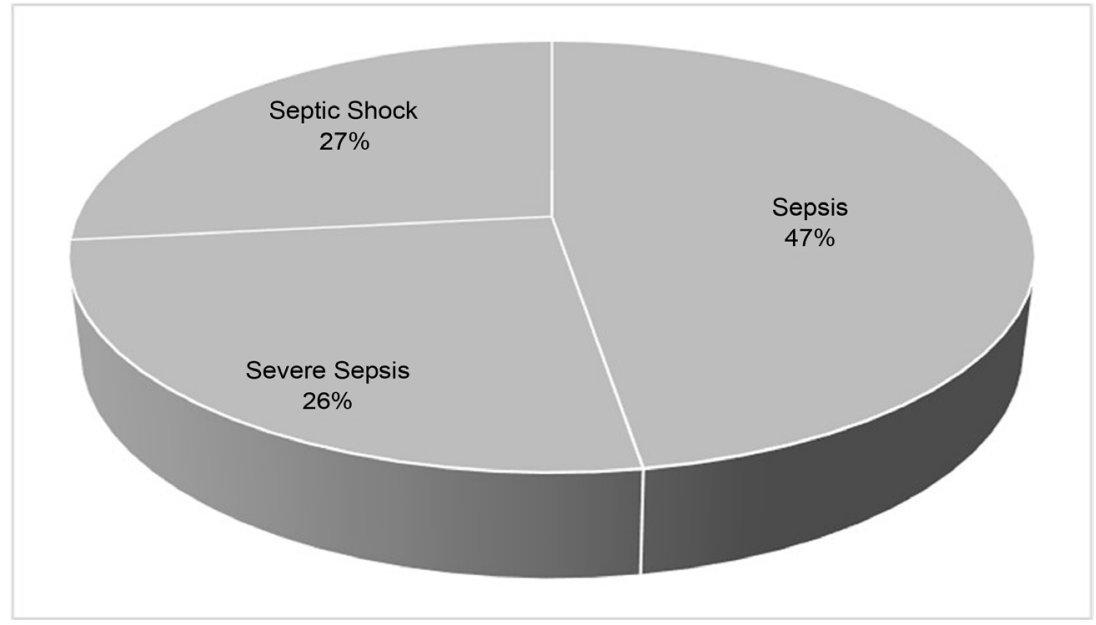

Figure 1. Percentage of patients with sepsis $(n=142)$, severe sepsis $(77)$ and septic shock (80).

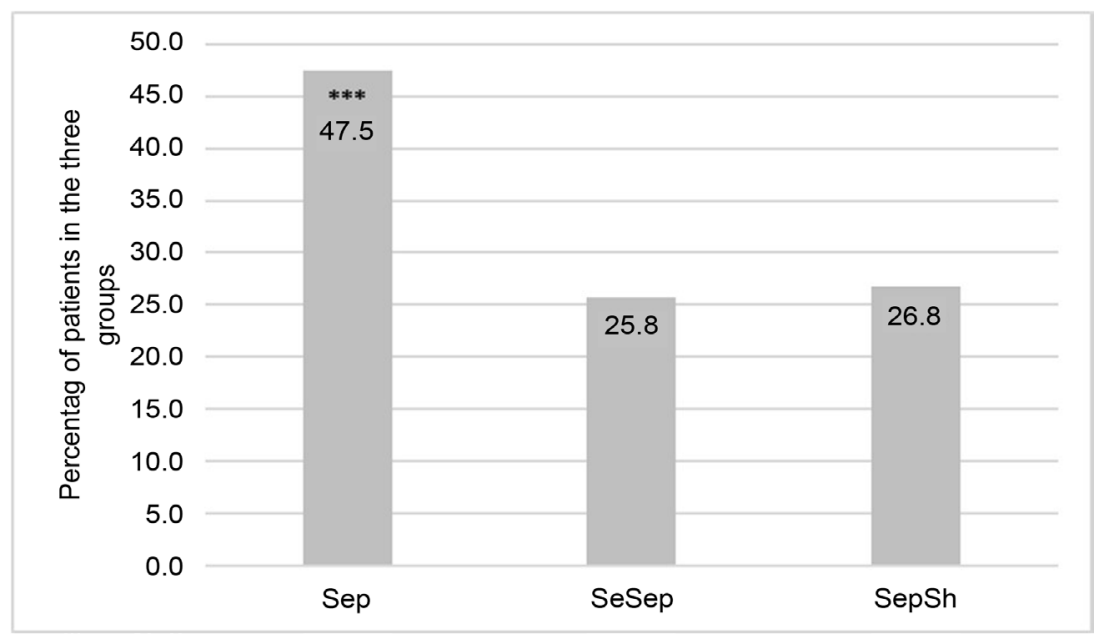

Figure 2. Distribution of patients between the septic, severely septic and septic shock groups. The number of patients in the septic group was significantly higher than in the other two $(\mathrm{p}<0.001)$.

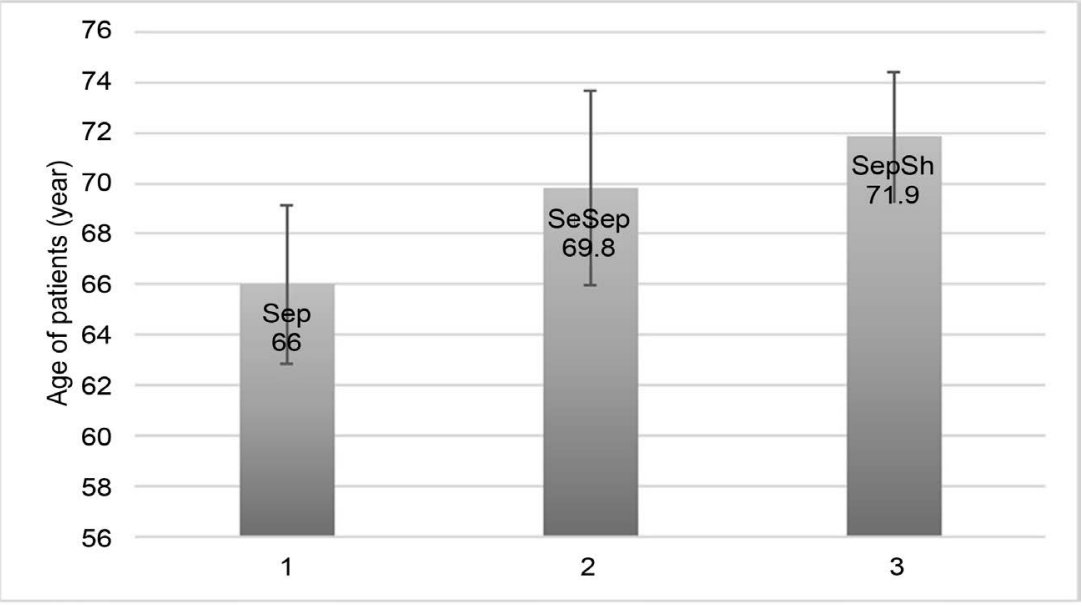

Figure 3. Age of patients with sepsis $(\mathrm{n}=142)$, severe sepsis $(77)$ and septic shock (80). Whiskers represent the $95 \%$ confidence intervals. 
Sep, $20.8 \%$ in the SeSep and $62.5 \%$ in the SepSh groups (Figure 5) reflecting significant difference when comparing the three groups. However there was no significant difference in the methods applied for invasive monitoring.

Blood cultures were drawn in $62(43.7 \%)$ patients with sepsis, 54 (70.1\%) with severe sepsis and $62(77.5 \%)$ in those with septic shock, showing significantly more samples in the severe sepsis and septic shock group compared to the patients with sepsis (Figure 6).

Table 2. Gender of patients in the three groups. The difference is not significant ( $\mathrm{p}=$ 0.496).

\begin{tabular}{cccc}
\hline & Sepsis & Severe sepsis & Septic shock \\
\hline \multirow{2}{*}{ Gender of patients } & $\delta 72(50.7 \%)$ & $\delta 46(59.7 \%)$ & $\precsim 43(53.8 \%)$ \\
& $\wp 70(49.3 \%)$ & $\wp 41(40.3 \%)$ & $\wp 37(46.2 \%)$ \\
\hline
\end{tabular}

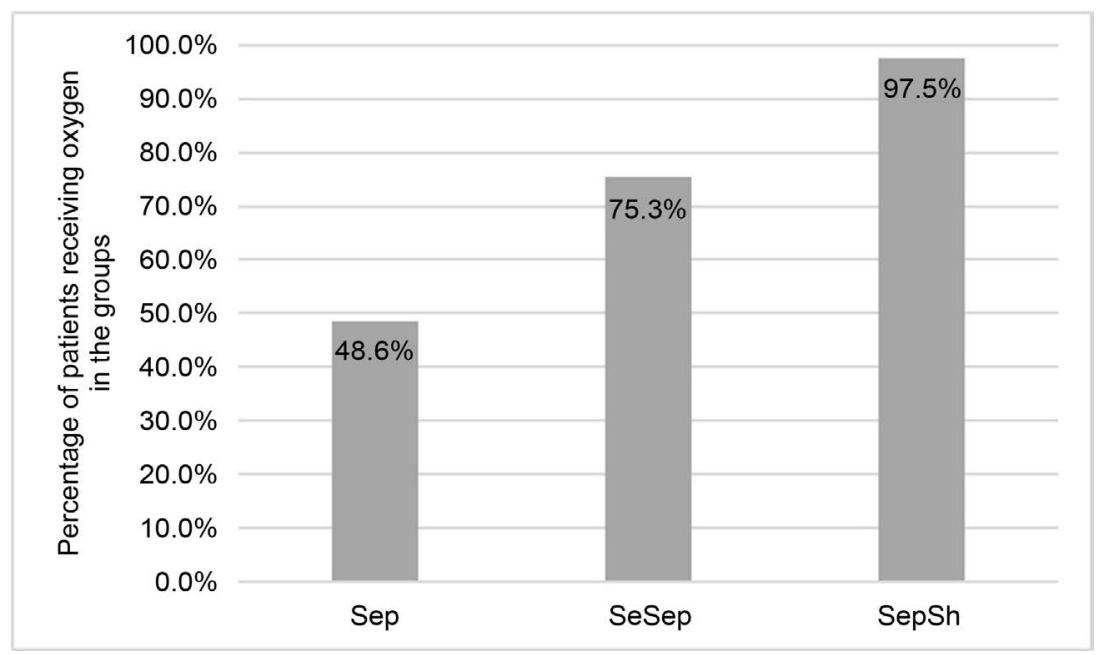

Figure 4. Percentage of patients receiving oxygen in the three groups. Significantly more patient received oxygen in the severely septic and septic shock groups compared to those suffering from sepsis $(\mathrm{p}<0.001)$.

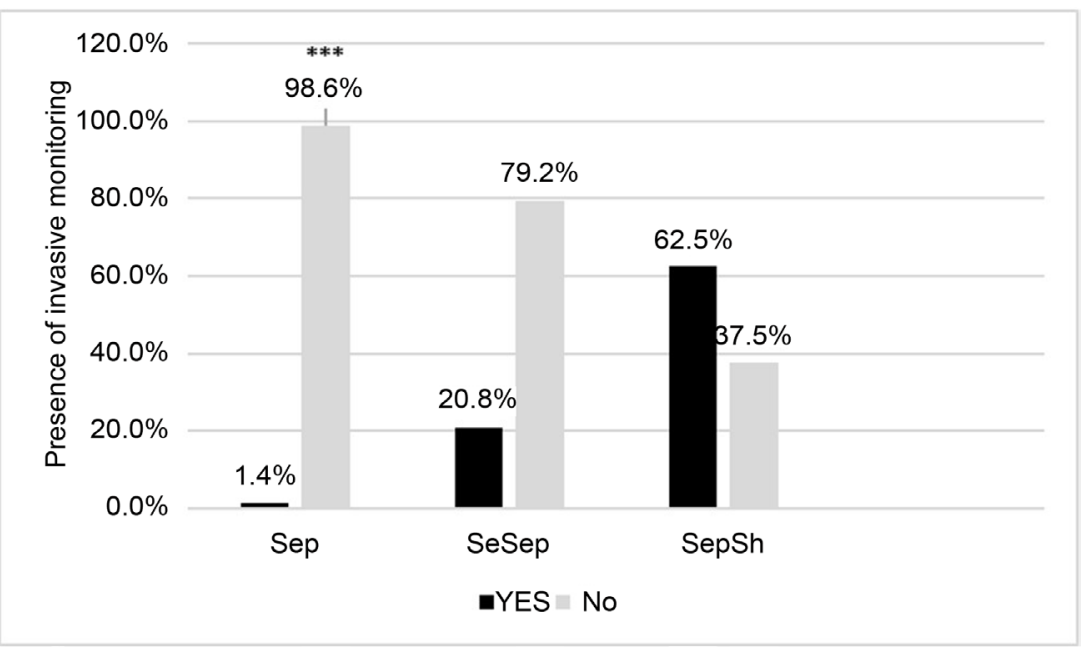

Figure 5. Proportion of invasive monitoring in the three patient groups. There were significantly less invasive measurements in the septic patients $(\mathrm{p}<0.001)$. 


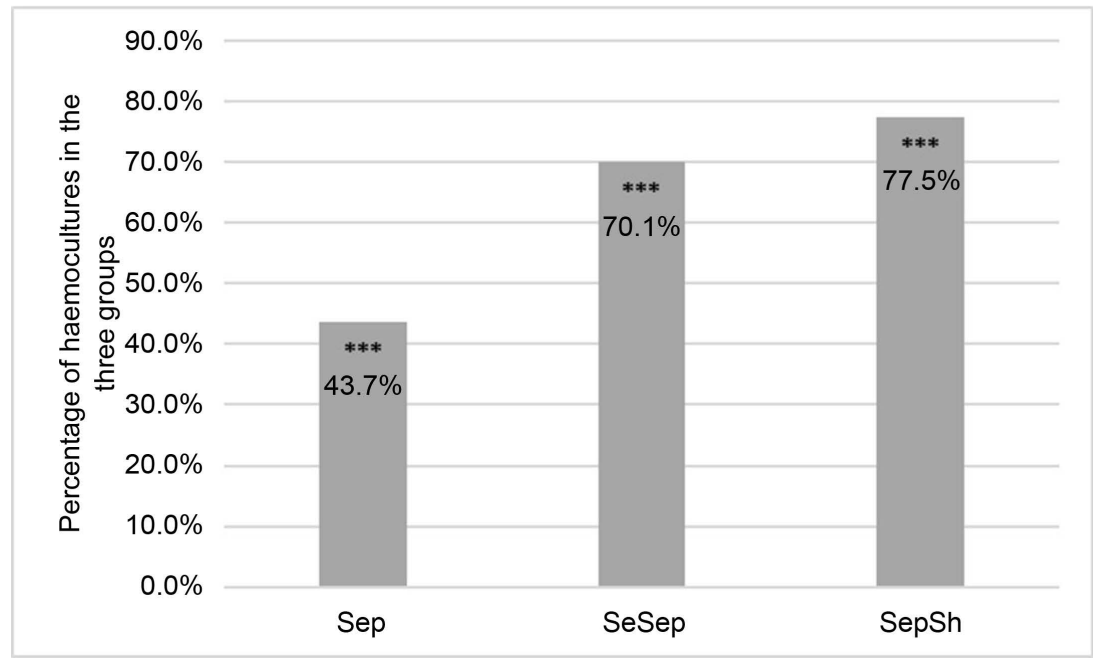

Figure 6. Proportion of haemoculture measurements in the septic, severely septic and septic shock patients. A significant difference was detected when comparing patients with severe sepsis and septic shock to those with sepsis $(\mathrm{p}<0.001)$.

With increasing severity more lactate measurements were carried out, 72 (50.7\%) in the septic, 45 (58.4\%) in the severely septic and 69 (86.3\%) in the septic shock patients, showing significant difference in the number of measurements (Figure 7(a)) and in terms of the absolute values of lactate (Figure 7(b)).

PCT was measured in 97 (68.3\%) septic, 60 (77.9\%) severely septic and 62 (77.5\%) septic shock patients (Figure 8(a)), with significant difference when comparing septic and severely septic or septic shock groups, but no significant difference was observed between those in the SeSep and SepSh groups (Figure 8(b)).

Administration of antibiotic was significantly more frequent in the SeSep and SepSh patients (Figure 9).

The time from arrival to antibiotic administration was 363 (SEM: 32) minutes in the septic, 228 (SEM: 25) minutes in the severely septic and 218 (SEM: 29) minutes in the septic shock patients showing significant difference between patients with severe sepsis, septic shock and those with sepsis. No significant difference was observed between the former two groups (Figure 10).

One-hundred and twenty-two (85.9\%) patients were disposed to general ward, $7(4.9 \%)$ to ICU, $6(4.2 \%)$ sent home, another $6(4.2 \%)$ to other institutions in the septic group. Forty-four (57.1\%) patients were accommodated in general ward, $23(29.9 \%)$ in intensive care, 8 (10.4\%) in other institutions. In the septic shock group 9 patients (11.3\%) were admitted to general ward, $49(61.3 \%)$ to ICU, 3 (3.8\%) to other institutions. Mortality was $0.7 \%, 2.6 \%$ and $23.8 \%$ in the septic, severely septic and septic shock patients, involving 1, 2, 19 patients, respectively (Figure 11) with disposition times of nearly the same duration (Figure 12). Admission to general ward, ICU was no different in the three groups along with mortality in the emergency department. Compared to the average distribution in the septic group significantly more patients were sent home and were disposed to general ward, and in the septic shock group significantly more patients were admitted to ICU or died. 


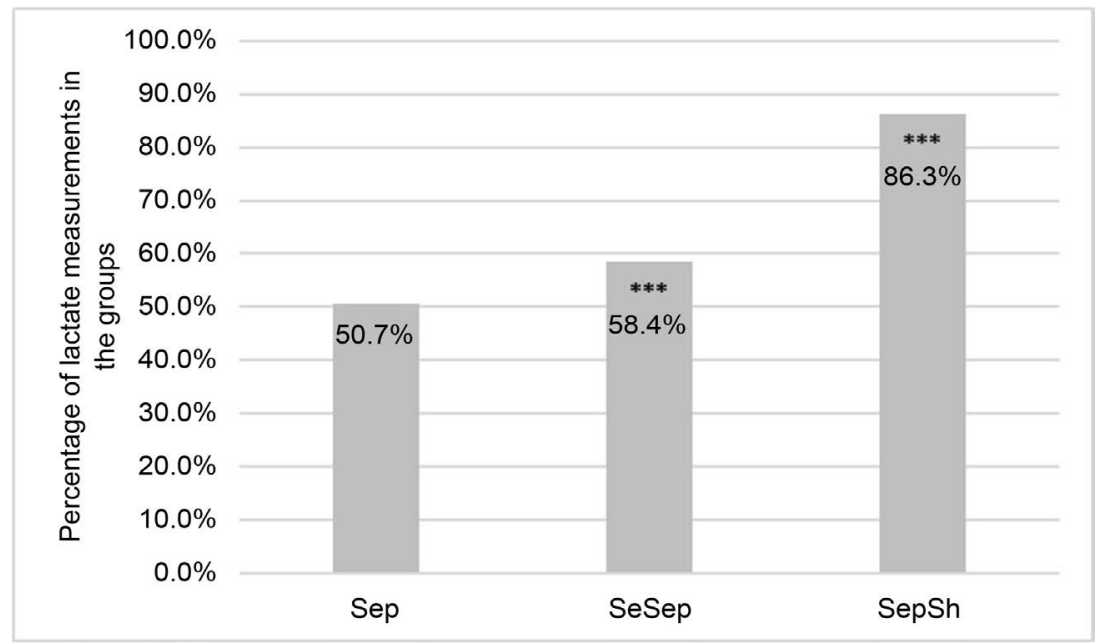

(a)

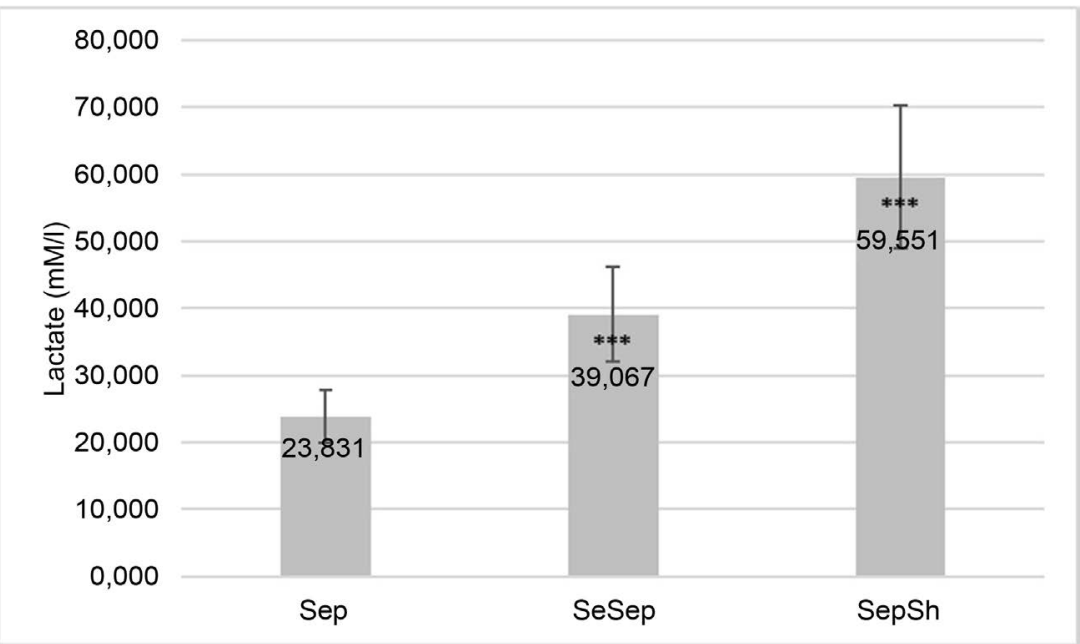

(b)

Figure 7. (a) Proportion of lactate measurements in the three groups. Significantly higher number of measurements were carried out with increasing severity $(\mathrm{p}<$ 0.001). (b) Absolute lactate values in the three different groups. Whiskers represent the $95 \%$ confidence interval. All values showed significant differences compared to each other $(\mathrm{p}<0.001)$.

\section{Discussion}

There is a growing body of evidence that timely management of sepsis decreases morbidity and mortality [16] [17] also contributing to the lowering of health care costs [18]. With the introduction of the Hungarian Emergency Sepsis Register we wanted to determine where and how we can improve our activity on the battlefield of sepsis in the front line.

It seems that sepsis is primarily the disease of the elderly population; however we received no data from paediatric emergency departments. The reason for this is that paediatric emergency care is separated in space in Hungary, even if emergency admissions take place at the same gate. It seems that regarding the examined population there is no age or gender related severity in sepsis according to our data. 


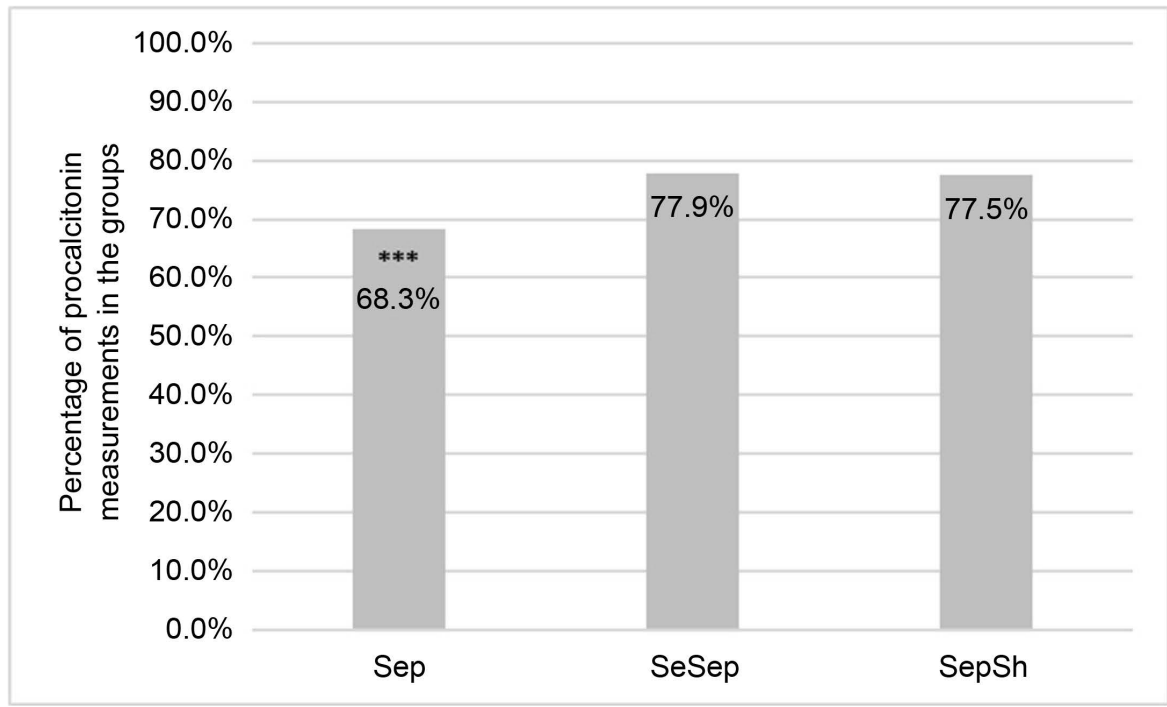

(a)

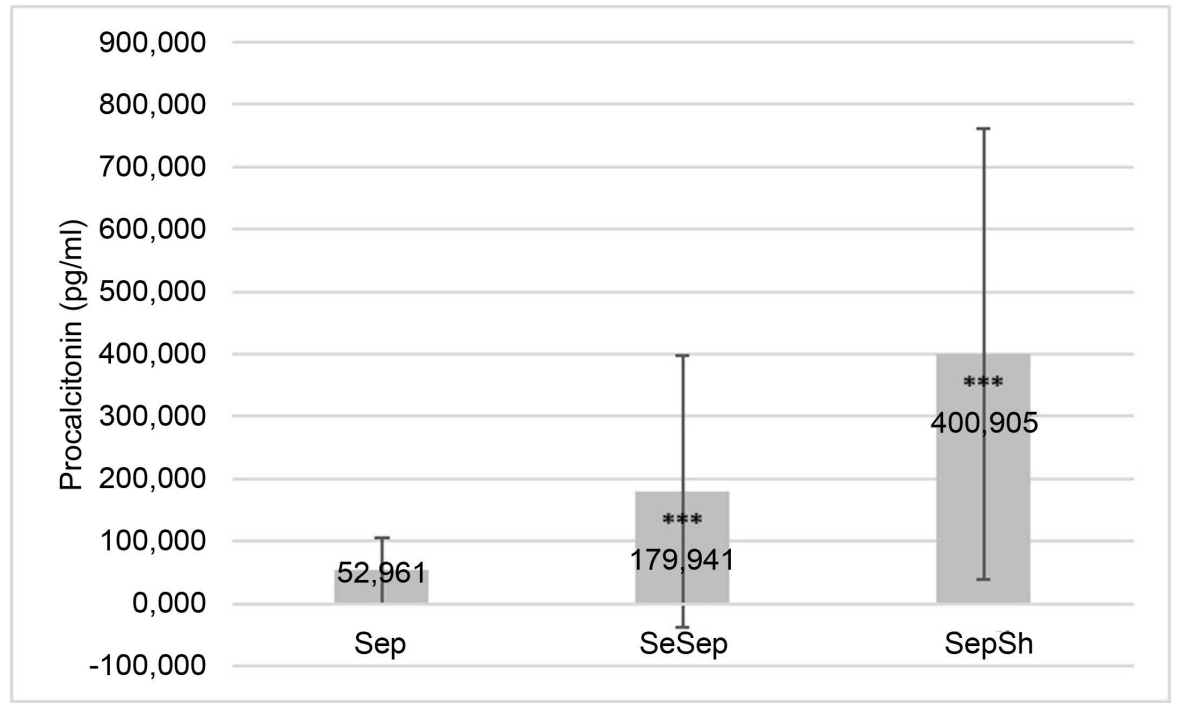

(b)

Figure 8. (a) Proportion of procalcitonin measurements in the three groups. Significantly higher number of measurements were carried out comparing the SeSep and SepSh groups to the Sep group, but there was no significant difference between SeSep and SepSh patients. (b) Absolute procalcitonin values in the three different groups. Whiskers represent the $95 \%$ confidence interval. All values showed significant differences compared to each other $(\mathrm{p}<0.001)$.

There is a clear correlation between severity of sepsis and administration of oxygen along with the involvement of invasive monitoring, such as arterial blood pressure, central venous pressure and oxygen saturation measurement; however, combined invasive monitoring is not applied frequently. There is an ongoing debate on the study of Rivers and his colleagues [7] regarding the usefulness of target values applied by them. It is widely believed that tissue perfusion and oxygenation are the key factors in the management of shock states, including sepsis; however, there are still unanswered questions about the most useful and optimal target parameters and values [19] [20] [21]. 


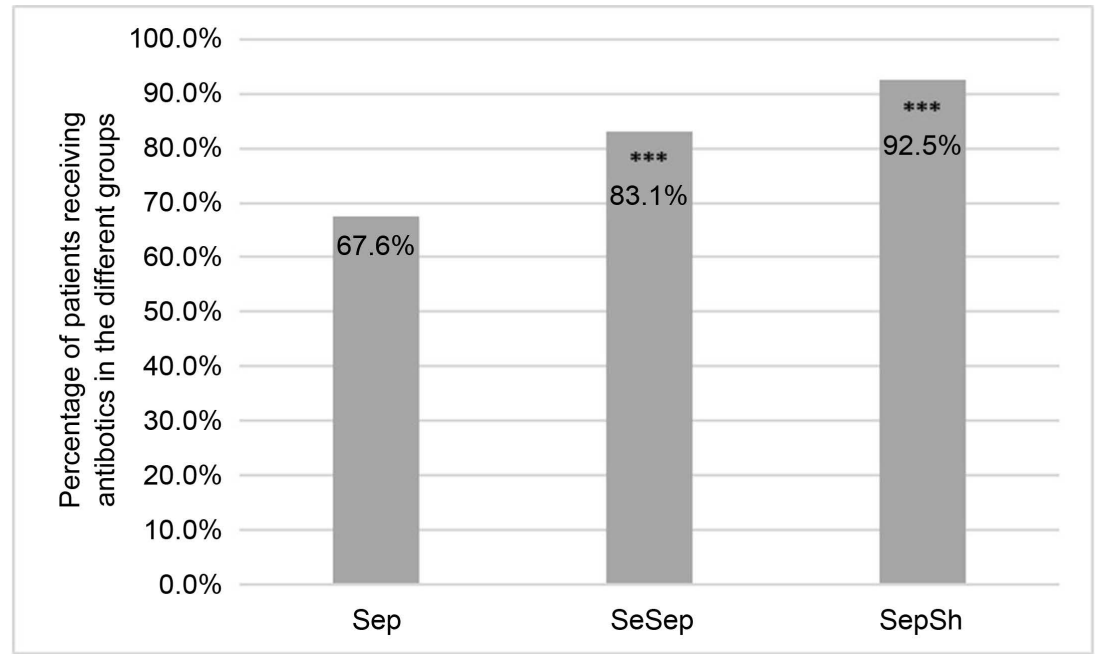

Figure 9. Proportion of patients receiving antibiotics in the three groups of septic, severely septic and septic shock patients. A significant difference was detected with increasing disease severity $(\mathrm{p}<0.001)$.

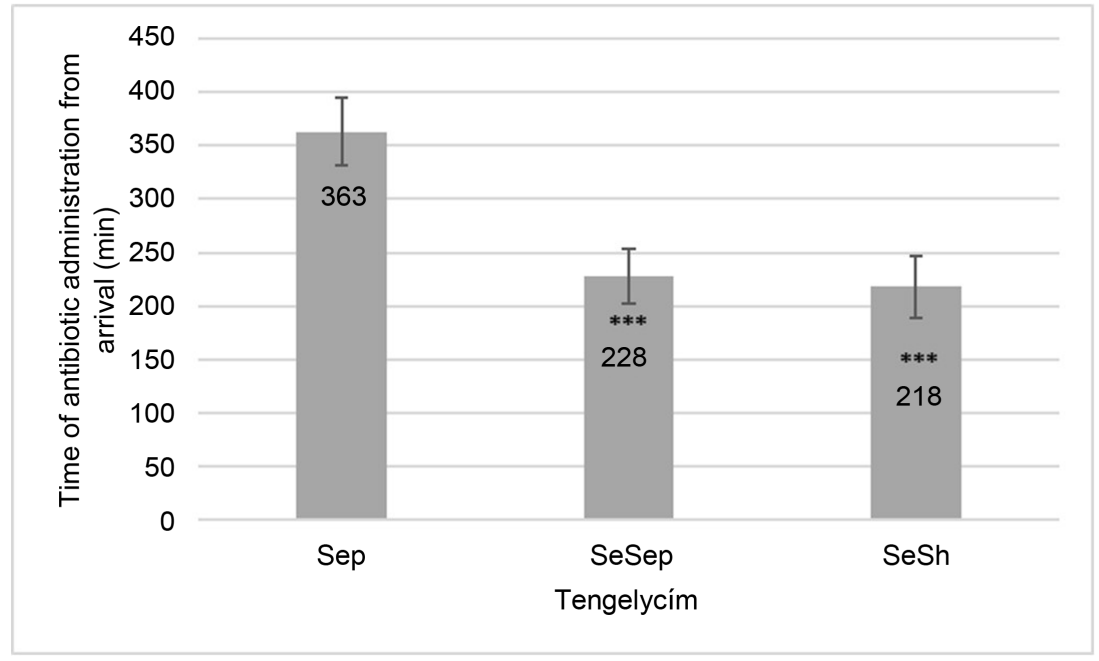

Figure 10. Time elapsed between arrival to the ED and antibiotic administration. A significant difference was detected in the severely septic and septic shock patients compared to those with sepsis $(\mathrm{p}<0.001)$, but no significant difference was seen in the former two. Whiskers represent the standard errors of mean.

It seems to be rather obvious that early detection, early goal directed therapy and early adequate referral should be based on actions that can easily be remembered and reproduced. The newest guidelines recommend an even simpler approach to identify septic patients and those with septic shock. Unfortunately emergency patients are not addressed fully by the new recommendations of SEPSIS-3; however an average of $40 \%$ of all subjects are coming from the emergency departments. It might be worth reckoning the need for a more detailed classification system that also encounters those patients who were classified as severely septic in the ED, along with those presenting with blood stream infection. As it is noted in the new recommendations, one of the limitations of the study was that only patients who had prior suspicion or confirmation of infection 


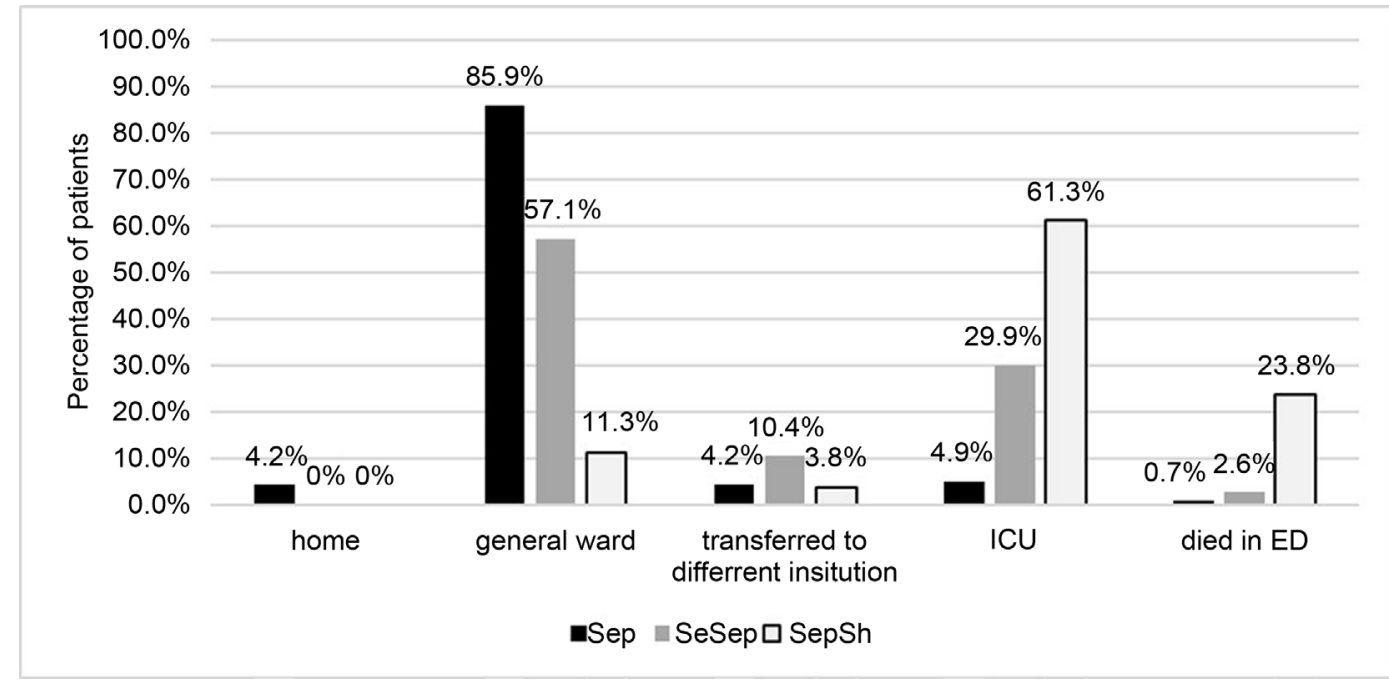

Figure 11. Disposition of patients to different locations. Significant differences were detected in the septic patients in terms of discharge to home and admission to general wards and also a significant difference was detected in those with septic shock regarding admittance to ICU and mortality in the ED.

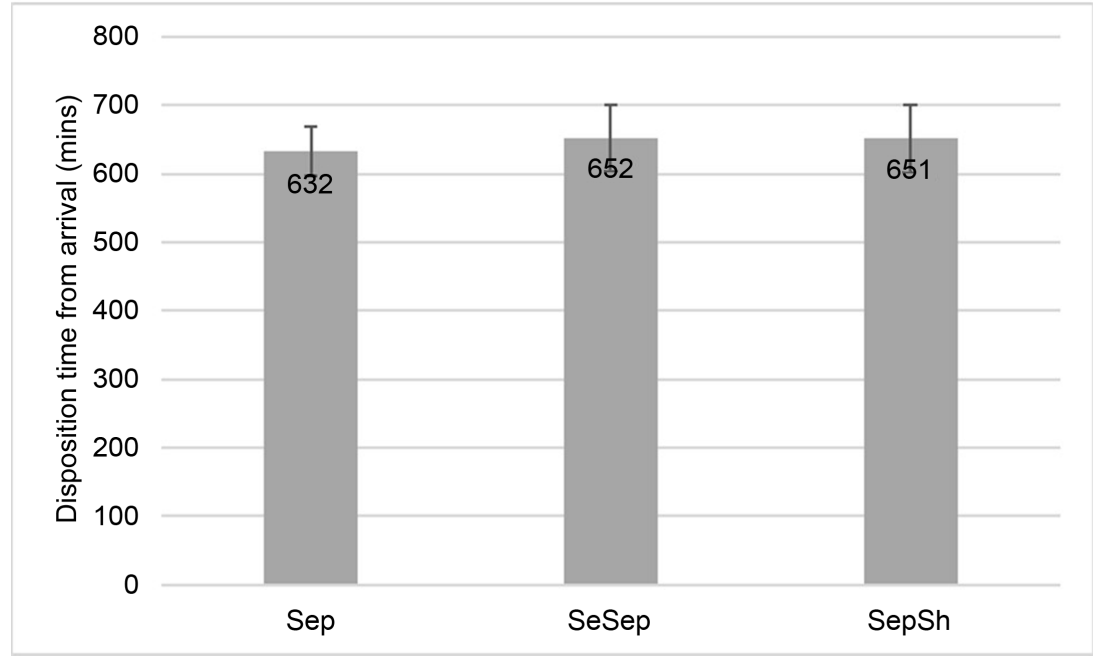

Figure 12. Disposition times in the three patient groups. No significant difference was detected. Whiskers represent the standard error of mean.

were included. Patients presenting in the ED are not "labelled" to have infection and the identification and diagnosis of sepsis still remain a challenge. Individual differences will remain to be key factors in the development of the septic process and will challenge the ED staff.

It is not surprising that lactate is not involved in the diagnosis of sepsis. There has been an ongoing debate on the usefulness of lactate as a biomarker of tissue hypoperfusion due to several concerns [15].

This issue is supported by our finding that lactate in septic patients was only mildly elevated; however, lactate correlates with the severity of the disease along with procalcitonin. It was rather surprising that procalcitonin was used more frequently than lactate in all three groups of patients. This might suggest that procalcitonin is thought to be a better sepsis marker for the Hungarian emer- 
gency physicians than lactate.

It is a question how swiftly the quick SOFA and SOFA scores can be adopted in the environment of the EDs, but the available calculators are already operating with these scoring systems. But it seems that Mortality in Emergency Department in Sepsis (MEDS) score is used more frequently than the recommended qSOFA and SOFA scores [22].

There is a reduction in morbidity and mortality with the right actions and one can see an improvement in the attitude of the acute caregivers when using target values such as mean arterial pressure, lactate either in patient classification or in therapy [23].

Our results reveal that sepsis is recognized as a problem in the Hungarian emergency departments. Classification was based on the internationally recognized guidelines [3] and lactate and procalcitonin were used as biomarkers of sepsis. It is reassuring that patients with severe sepsis and septic shock received more attention in terms of monitoring and biomarker analysis. This was also reflected in oxygen administration, while only some of the septic patients received additional oxygen, severely septic patients and those in septic shock were given oxygen in increasing number.

The biggest issue was the administration of antibiotics to our patients in the emergency department along with the delay of administration. The small percentage in the septic patient group receiving antibiotics is further complicated by the fact that only a few of our patients received antibiotics within the recommended time (i.e. 60 minutes from arrival); however the time from arrival to oxygen administration shortened with disease severity.

It is worth noting that many EDs in Hungary have ICU capabilities and capacities, therefore a high number of patients could be kept in the ED for 24 hours where intensive therapy could be initiated.

One of the weaknesses of the questionnaire was that there was no information on ICU capacities in the EDs involved in this study, therefore we can only speculate on the level of treatment of patients requiring critical care.

Initially it was surprising to see that a few patients were sent home with the diagnosis of sepsis. Strictly speaking and observing the Surviving Sepsis Campaign criteria [3] any patient with leukocytosis, fever and a diagnosed with pneumonia classified as septic. Using appropriate scores and risk assessmentas part of standard procedures in the emergency wards-allowed us to decide on the need of hospitalization and therefore sepsis per se does not require hospital admission in all cases; however a critical judgment must be made considering may factors to discharge these patients and organize the appropriate follow-up.

Another weakness of this data collection was that categorization of patients was the discretion of the acute care professional. Sepsis is a dynamic process, deterioration can happen within a short period of time. Considering the long wait times which is not unique in Hungary but very true in other parts of the developed world, one cannot be absolutely sure where his or her patient belongs to in terms of severity of sepsis over the time in the ED. 
The pattern of patients' disposition in the three studied groups requires explanation. While it is quite understandable, that those who presented with uncomplicated sepsis were mostly discharged to general wards, it is perplexing, that more than half of the patients with septic organ failure and one tenth of those with shock were also disposed to these facilities. One has to keep in mind that in Hungary there are no formal network of hospice services and the majority of our hospitals lack these units. Therefore patient presenting with incurable conditions are often admitted to general hospital wards. This factor may accounts for the unexplained patient dispositions. Other observations, e.g. the length of stay at the ED might be also distorted by the same factor. Duration of hospitalization and the ever increasing costs of treating septic patients is also an important aspect. In this survey we had no information on the hospital stay since the data collection focused on the ED activity but it is also a challenging task to look into financial issues, i.e. how are these affected by early recognition and early treatment in the ED.

Nevertheless, this sepsis register is a good start point not only in valid and focused data collection, but also in a mind-set of the Hungarian emergency physicians-most importantly to improve the recognition of sepsis, shorten the times of antibiotic administration and appropriate discharge routes from the ED. There is an ongoing education in sepsis recognition and treatment in the prehospital and also in the intrahospital setting targeting better early recognition and early treatment. The four medical universities and the Hungarian Society of Emergency Medicine signed a joint agreement on education of medical staff dealing with septic patients, with special focus on the SEPSIS 6 approach [10] [11] in all emergency departments who joined to the move on sepsis recognition. It is also prudent to make clear that investing in early detection and early treatment will have an impact not only on survival but also on cost. The new questionnaire and data collection will focus on the achievements of the joint education.

Understanding that public education is also a key point in early recognition therefore we joined the World Sepsis Day campaign with national events that are open to anyone who would like to help in the fight against sepsis [24].

\section{Acknowledgements}

The authors would like to thank the invaluable contribution in data collection of Dr. Péter Arányi, Dr. Gerda Lóczi, Dr. Zsófia Jordán and Dr. Tamás Nagy. We would also like to thank the professional assistance of Professor László Rudas.

\section{References}

[1] Reinhart, K., Daniels, R., Kissoon, N., et al. (2013) GSA Executive Board and WSD Executive Board. The Burden of Sepsis-A Call to Action in Support of World Sepsis Day 2013. Journal of Critical Care, 28, 526-528.

https://doi.org/10.1016/j.jcrc.2013.04.012

[2] Dellinger, R.P. (2015) The Surviving Sepsis Campaign: Where Have We Been and Where Are We Going? Cleveland Clinic Journal of Medicine, 82, 237-244. 
[3] Dellinger, R.P., Levy, M.M., Rhodes, A., et al. (2013) Surviving Sepsis Campaign Guidelines Committee including the Pediatric Subgroup. Surviving Sepsis Campaign: International Guidelines for Management of Severe Sepsis and Septic Shock: 2012. Critical Care Medicine, 41, 580-637. https://doi.org/10.1097/CCM.0b013e31827e83af

[4] Angus, D.C. and van der Poll, T. (2013) Severe Sepsis and Septic Shock. New England Journal of Medicine, 69, 840-851. https://doi.org/10.1056/NEJMra1208623

[5] Mearelli, F., Orso, D., Fiotti, N., et al. (2015) Sepsis Outside Intensive Care Unit: The Other Side of the Coin. Infection, 43, 1-11. https://doi.org/10.1007/s15010-014-0673-6

[6] Levy, M.M., Artigas, A., Phillips, G.S., et al. (2012) Outcomes of the Surviving Sepsis Campaign in Intensive Care Units in the USA and Europe: A Prospective Cohort Study. Lancet Infectious Diseases, 12, 919-924. https://doi.org/10.1016/S1473-3099(12)70239-6

[7] Rivers, E., Nguyen, B., Havstad, S., et al. (2001) Early Goal-Directed Therapy Collaborative Group: Early Goal-Directed Therapy in the Treatment of Severe Sepsis and Septic Shock. New England Journal of Medicine, 345, 1368-1377. https://doi.org/10.1056/NEJMoa010307

[8] Peake, S.L., Delaney, A., Bailey, M., et al., The ARISE Investigators (2014) GoalDirected Resuscitation for Patients with Early Septic Shock. New England Journal of Medicine, 371, 1496-1506. https://doi.org/10.1056/NEJMoa1404380

[9] Yealy, D.M., Kellum, J.A., Huang, D.T., et al., The PROCESS Investigators (2014) A Randomized Trial of Protocol-Based Care for Early Septic Shock. New England Journal of Medicine, 370, 1683-1693. https://doi.org/10.1056/NEJMoa1401602

[10] Daniels, R., Nutbeam, T., McNamara, G., et al. (2011) The Sepsis Six and the Severe Sepsis Resuscitation Bundle: A Prospective Observational Cohort Study. Emergency Medicine Journal, 28, 507-512. https://doi.org/10.1136/emj.2010.095067

[11] McGregor, C. (2014) Improving Time to Antibiotics and Implementing the "Sepsis 6". BMJ Quality Improvement Reports, 2, 1-3. https://doi.org/10.1136/bmjquality.u202548.w1443

[12] Adcroft, L. (2014) Improving Sepsis Management in the Acute Admissions Unit. BMJ Quality Improvement Reports, 3, 1-3. https://doi.org/10.1136/bmjquality.u204974.w2091

[13] Kumar, A., Roberts, D., Wood, K.E., et al. (2006) Duration of Hypotension before Initiation of Effective Antimicrobial Therapy Is the Critical Determinant of Survival in Human Septic Shock. Critical Care Medicine, 34, 1589-1596. https://doi.org/10.1097/01.CCM.0000217961.75225.E9

[14] Singer, M., Deutschman, C.S., Seymour, C.W., et al. (2016) The Third International Consensus Definitionsfor Sepsis and Septic Shock (Sepsis-3). JAMA, 315, 801-810. https://doi.org/10.1001/jama.2016.0287

[15] Suetrong, B. and Walley, K. (2016) Lactic Acidosis in Sepsis: It's Not All Anaerobic. Chest, 149, 252-261. https://doi.org/10.1378/chest.15-1703

[16] Amland, R.C., Lyons, J.J., Greene, T.L. and Haley, J. (2015) A Two-Stage Clinical Decision Support System for Early Recognition and Stratification of Patients with Sepsis: An Observational Cohort Study. JRSM Open, 6, 1-10. https://doi.org/10.1177/2054270415609004

[17] Amland, R.C., Haley, J.M. and Lyons, J. (2015) A Multidisciplinary Sepsis Program Enabled by a Two-Stage Clinical Decision Support System: Factors That Influence Patient Outcomes. American Journal of Medical Quality, 31, 501-508. 
https://doi.org/10.1177/1062860615606801

[18] Jones, S.L., Ashton, C.M., Kiehne, L., et al. (2015) Reductions in Sepsis Mortality and Costs after Design and Implementation of a Nurse-Based Early Recognition and Response Program. Joint Commission Journal on Quality and Patient Safety, 41, 483-491. https://doi.org/10.1016/S1553-7250(15)41063-3

[19] Yealy, D.M., Kellum, J.A., Huang, D.T., et al. (2014) Process Investigators. A Randomized Trial of Protocol-Based Care for Early Septic Shock. New England Journal of Medicine, 370, 1683-1693. https://doi.org/10.1056/NEJMoa1401602

[20] Peake, S.L., Delaney, A., Bailey, M., et al., ARISE Investigators (2014) ANZICS Clinical Trials Group. Goal-Directed Resuscitation for Patients with Early Septic Shock. New England Journal of Medicine, 371, 1496-1506. https://doi.org/10.1056/NEJMoa1404380

[21] Mouncey, P.R., Osborn, T.M., Power, G.S., et al. (2015) ProMISe Trial Investigators. Trial of Early, Goal-Directed Resuscitation for Septic Shock. New England Journal of Medicine, 372, 1301-1311. https://doi.org/10.1056/NEJMoa1500896

[22] Williams, J.M., Greenslade, J.H., Chu, K., et al. (2016) Severity Scores in Emergency Department Patients with Presumed Infection: A Prospective Validation Study. Critical Care Medicine, 44, 539-547. https://doi.org/10.1097/CCM.0000000000001427

[23] Burrell, A.R., McLaws, M., Fullick, M., et al. (2016) SEPSIS KILLS: Early Intervention Saves lives. Medical Journal of Australia, 2, 1e1-1e7.

[24] http://www.world-sepsis-day.org/

Submit or recommend next manuscript to SCIRP and we will provide best service for you:

Accepting pre-submission inquiries through Email, Facebook, LinkedIn, Twitter, etc. A wide selection of journals (inclusive of 9 subjects, more than 200 journals)

Providing 24-hour high-quality service

User-friendly online submission system

Fair and swift peer-review system

Efficient typesetting and proofreading procedure

Display of the result of downloads and visits, as well as the number of cited articles

Maximum dissemination of your research work

Submit your manuscript at: http://papersubmission.scirp.org/

Or contact ojepi@scirp.org 